\title{
Upregulation of miR-194 contributes to tumor growth and progression in pancreatic ductal adenocarcinoma
}

\author{
JING ZHANG $^{1 *}$, CHEN-YAN ZHAO $^{1 *}$, SHU-HUI ZHANG ${ }^{2 *}$, DANG-HUI YU ${ }^{1}$, YING CHEN $^{1}$, \\ QING-HUA LIU ${ }^{1}$, MIN SHI ${ }^{1}$, CAN-RONG NI ${ }^{1}$ and MING-HUA ZHU ${ }^{1}$ \\ ${ }^{1}$ Department of Pathology, Changhai Hospital, Second Military Medical University, Shanghai 200433; \\ ${ }^{2}$ Department of Pathology, Yueyang Hospital, Shanghai University of Traditional \\ Chinese Medicine, Shanghai 200437, P.R. China
}

Received October 17, 2013; Accepted December 5, 2013

DOI: $10.3892 / o r .2013 .2960$

\begin{abstract}
Pancreatic ductal adenocarcinoma (PDAC) is one of the most lethal types of human cancer worldwide. In the present study, we investigated the diagnostic and biological significance of microRNA-194 (miR-194) in PDAC. miRNA expression profiling of human PDACs and adjacent normal pancreatic tissues identified a total of 16 genes including miR-194 with $>1.15$-fold expression changes $(8$ overexpressed and 8 underexpressed). Quantitative real-time polymerase chain reaction (PCR) revealed elevation of serum miR-194 levels were significantly greater in PDAC patients than in duodenal adenocarcinoma patients and healthy controls. Receiver operating characteristic analysis demonstrated that serum miR-194 had a sensitivity of $54.3 \%$ and a specificity of $57.5 \%$ for discriminating PDAC patients from healthy controls. Combined analysis of the 3 groups yielded a sensitivity of 84.0 and a specificity of $75.0 \%$ for the combined detection of miR-192 and miR-194 in the diagnosis of PDAC. Ectopic expression of miR-194 in PANC-1 pancreatic cancer cells enhanced cell proliferation, migration and colony formation, which was coupled with decreased expression of the tumor suppressor DACH1. miR-194 overexpression increased tumor growth and local invasion and suppressed the expression of DACH1 in an orthotopic pancreatic cancer mouse model. In conclusion, upregulation of miR-194 contributes to tumor growth and progression in PDAC, possibly through suppression of DACH1. However, serum miR-194 has a low capacity for detection of PDAC. Combined detection of serum miR-192 and miR-194 levels may serve as a sensitive diagnostic biomarker for PDAC.
\end{abstract}

Correspondence to: Professor Ming-Hua Zhu, Department of Pathology, Changhai Hospital, Second Military Medical University, 168 Changhai Road, Shanghai 200433, P.R. China

E-mail:mhzhu2000@hotmail.com; mhzhu07@126.com

*Contributed equally

Key words: pancreatic ductal adenocarcinoma, microRNA, diagnosis, growth, progression, targets

\section{Introduction}

Pancreatic ductal adenocarcinoma (PDAC) ranks among the most lethal types of human cancer, with a 5-year survival rate of $<5 \%$ and a median survival time of $<6$ months $(1,2)$. Late presentation and aggressive metastatic progression are important factors contributing to the extremely poor prognosis of PDAC. Several non-invasive imaging techniques such as magnetic resonance imaging have been used to detect PDAC. However, early diagnosis of this disease remains clinically challenging, as currently available imaging techniques fail to reliably detect pancreatic tumors $<1 \mathrm{~cm}$ in size (3). Therefore, identification of novel molecular targets for early diagnosis and therapeutic intervention is of importance for clinical management of this malignancy.

MicroRNAs (miRNAs) are a large family of non-coding, single-stranded RNAs of 22 nucleotides, which negatively regulate gene expression by binding to the 3 '-untranslated region (UTR) of target mRNAs, leading to accelerated mRNA turnover and/or translational repression (4). In a canonical pathway, miRNAs are expressed as primary miRNA transcripts, which are cleaved into pre-miRNA molecules, exported from the nucleus, and further processed by Dicer to produce mature miRNAs. Compelling evidence indicates that miRNAs actively participate in carcinogenesis, acting as oncogenes or tumor suppressor genes (5); they can simultaneously regulate a large number of target genes and affect many aspects of tumor progression such as proliferation, migration, invasion, epithelial-mesenchymal transition and angiogenesis $(6,7)$. Given that miRNAs are frequently deregulated in cancer and can be stably detected in human plasma or serum, circulating miRNAs are increasingly being explored as noninvasive diagnostic biomarkers for early cancer detection (8).

Many individual miRNAs have been implicated in the pathogenesis of PDAC. For instance, Liffers et al (9) reported that miR-148a is downregulated in PDACs and negatively regulates tumor cell growth and colony formation. miR-21 was found to be required for PDAC cell proliferation and survival, as its downregulation led to reduced cell proliferation and increased cell death in MIA PaCa-2 PDAC cells (10). The diagnostic values of circulating miRNAs have been described in pancreatic cancer. Liu et al (11) proposed that a panel of 
7 miRNAs (i.e., miR-20a, miR-21, miR-24, miR-25, miR-99a, miR-185 and miR-191) have high sensitivity and specificity for distinguishing pancreatic cancer from cancer-free controls and from chronic pancreatitis. The application of miRNA profiling technology has made great progress in developing novel biomarkers and therapeutic targets for PDAC. By combining tissue microdissection with global miRNA array analyses, Munding et al (12) identified miR-135b as an effective biomarker for PDAC, which can discriminate chronic pancreatitis from PDAC with a sensitivity and specificity of $>90 \%$.

In the present study, we also employed microarray techniques to identify novel differentially expressed miRNAs and tested their potential as diagnostic biomarker for PDAC. Additionally, the biological functions of miRNAs of interest in PDAC development and progression were investigated.

\section{Materials and methods}

Patients and samples. For miRNA profiling analysis, 9 cases of PDAC and 3 adjacent non-tumorous pancreatic tissues were obtained from 9 PDAC patients ( 4 males and 5 females, aged 47-75 years) who underwent surgical resection of tumors at the Department of Pancreatic Surgery of Changhai Hospital (Shanghai, China). For measurement of the expression of miRNAs of interest, 31 pairs of freshly resected PDACs and their corresponding non-tumorous tissues were collected from a second cohort of patients with PDAC (15 males and 16 females, aged 45-77 years, with a median age of 61 years). For assessment of the diagnostic significance of serum miRNAs, we enrolled an independent cohort of 70 PDAC patients, 17 age-matched duodenal adenocarcinoma patients, and 40 age-matched healthy controls. All PDAC patients were pathologically diagnosed by biopsy, and those with any prior anticancer therapy were excluded from the present study. All procedures involving humans (ref. no. CTcp008) were approved by the Ethics Committee of the Second Military Medical University (Shanghai, China) in accordance with the Helsinki Declaration and written informed consent was obtained from all patients. All animal experiments were undertaken in accordance with the National Institutes of Health Guide for the Care and Use of Laboratory Animals, with the approval of the Scientific Investigation Board of the Second Military Medical University (no. Lacp0010).

Cells and culture. Six pancreatic cancer cell lines, SW1990, BxPC-3, MIA Paca-2, PANC-1, Capan-1 and AsPC-1, were obtained from American Type Culture Collection (Manassas, VA, USA). SW1990, AsPC-1, BxPC-3 and PANC-1 cells were cultured in RPMI-1640 medium supplemented with $10 \%$ fetal bovine serum (FBS) (PAA, Vienna, Austria), MIA PaCa-2 cells in Dulbecco's modified Eagle's medium (DMEM) supplemented with 10\% FBS and 2.5\% horse serum (PAA), and Capan-1 cells in Iscove's modified Dulbecco's medium (IMDM) containing 20\% FBS. All cultures were maintained at $37^{\circ} \mathrm{C}$ in a $5 \%(\mathrm{v} / \mathrm{v}) \mathrm{CO}_{2}$ humidified atmosphere.

For examination of miRNA expression and secretion, the pancreatic cancer cells were seeded onto 6-well plates at a density of $2 \times 10^{6}$ cells/well. After a $24-\mathrm{h}$ incubation in serum-free media, the cells and their culture supernatants were separately collected and subjected to quantitative real-time polymerase chain reaction (qRT-PCR) analysis.

miRNA microarray analysis. miRNA expression profile analysis was performed using an Agilent miRNA microarray platform (Agilent, Santa Clara, CA, USA) that can simultaneously measure 713 miRNAs (13). Total RNA was extracted from human PDACs and adjacent normal pancreatic tissues using a mirVana PARIS kit (AM1561; Ambion, Austin, TX, USA) according to the manufacturer's instructions and as previously described (14). Cy3 labeling of RNA was performed using a miRNA labeling reagent and hybridization kit (Agilent 5190-0408; Agilent, Palo Alto, CA, USA). An Agilent microarray scanner and Agilent feature extraction software were employed for microarray data collection and analysis. Microarray scanning and data acquisition were performed at the Shanghai Biochip Company (Shanghai, China). Raw microarray data were normalized using the variance stabilization and normalization method. A two-sample t-test was used for the statistical analysis and a P-value $<0.05$ was considered statistically significant. In each group, the proportion of samples with a detection value of 1 was required to be $>60 \%$.

Locked nucleic acid-based in situ hybridization (LNA-ISH). LNA-ISH was performed using LNA $^{\mathrm{TM}}$ probes against miR-194 (Exiqon, Vedbaek, Denmark) on 5 frozen human PDAC samples according to the manufacturer's protocol, as previously described $(14,15)$.

miRNA expression detected by $q R T-P C R$. Total RNA was extracted from tissues or cultured cells using the mirVana PARIS kit, as described above. Reverse transcription reaction was carried out using a TaqMan MicroRNA Reverse Transcription kit (Applied Biosystems, Foster City, CA, USA). miRNA concentrations were quantified in duplicate by real-time PCR using a human TaqMan MicroRNA Assay kit (Applied Biosystems). Cycle threshold $(\mathrm{Ct})$ values were calculated using SDS 1.4 software (Applied Biosystems). The relative expression of miRNAs was calculated with the $2^{-\Delta \Delta C t}$ method and normalized against U6 snRNA (16).

Plasmids and preparation of lentiviral particles. The pGCLV-miR-194 plasmid, which expresses full-length human pre-miR-194 and a control empty plasmid were purchased from Shanghai Jikai Gene Chemical Technology (Shanghai, China). For production of lentiviral particles expressing miR-194, 293T cells were transfected with the expression vector pGCLV-miR-194 or control plasmid along with the packaging vectors using Lipofectamine ${ }^{\circledR} 2000$ (Invitrogen, Carlsbad, CA, USA), according to the manufacturer's instructions. The recombinant lentiviral particles were collected and purified. The multiplicity of infection (MOI) was determined in 293T cells. Aliquots of lentivirus were stored at $-80^{\circ} \mathrm{C}$ until use.

Transduction of lentiviral particles. For transduction of recombinant lentiviral particles, PANC-1 cells were seeded onto 6-well plates at a density of $3 \times 10^{5}$ cells/well and incubated overnight in RPMI-1640 medium with 10\% FBS. Due to the low endogenous levels of miR-194, PANC-1 cells were chosen for gain-of-function experiments. PANC-1 cells were 
then transduced with lentiviral particles at an MOI of 20 in the presence of polybrene $(6 \mu \mathrm{g} / \mathrm{ml})$. Cells were refreshed with culture medium $24 \mathrm{~h}$ later. Since the $\mathrm{pGC}-\mathrm{LV}$ vector expresses green fluorescent protein (GFP), GFP-positive cells were sorted by flow cytometry and expanded. qRT-PCR analysis was carried out to confirm the overexpression of miR-194 in the transduced PANC-1 cells.

MTT assay. Non-transduced and transduced PANC-1 cells were plated in triplicate in 96-well microplates at a density of $1.5 \times 10^{3}$ cells/well. Cell viability was assessed once daily for 6 consecutive days after seeding, using the 3-(4,5-dimethylthiazol-2-yl)-2,5-diphenyltetrazolium bromide (MTT) assay. MTT solution (5 g/l; Sigma, St. Louis, MO, USA) was added to the cell cultures and incubated at $37^{\circ} \mathrm{C}$ for $4 \mathrm{~h}$. After removal of the MTT solution, dimethyl sulfoxide solution was added to dissolve the formazan crystals. The absorbance was measured at a wavelength of $490 \mathrm{~nm}$. The experiments were repeated 3 times. Growth curves were constructed by plotting the absorbance values vs. time.

Colony formation assay. Non-transduced and transduced PANC-1 cells were plated onto 6-well plates at a density of 4,200 cells/well and incubated for 10 days at $37^{\circ} \mathrm{C}$. Cells were washed, fixed in $10 \%$ methanol for $15 \mathrm{~min}$ and stained in Giemsa for 10-30 min. Colonies consisting of $>50$ cells were counted under a low-magnification microscope. The experiments were repeated 3 times. Colony formation rate was calculated as colony number/total cells seeded x $100 \%$.

In vitro wound healing assay. Transduced PANC-1 cell migration was determined using an in vitro wound healing assay. Briefly, cells were seeded onto 6-well plates at a density of $5 \times 10^{5}$ cells/well. After incubation for $48 \mathrm{~h}$, an open area or 'scratch' was produced in the cell monolayers using a $100 \mathrm{ml}$ pipette tip. The cell cultures were washed several times to remove the displaced cells in the open area and then incubated for $48 \mathrm{~h}$. The extent of wound closure was examined by phase-contrast microscopy.

Tumorigenicity in nude mice. Nude mice were purchased from the Experimental Animal Center of Shanghai (Shanghai, China) and randomly divided into 2 groups $(n=4)$. To establish orthotopic pancreatic tumors, animals were anesthetized and the pancreas was exposed via an abdominal incision. The mice were injected into the pancreas at a single site with $5 \times 10^{7}$ miRNA-194-overexpressing or empty vector-transduced PANC-1 cells. After tumor cell implantation, the pancreas was carefully returned to the peritoneal cavity and the abdomen was closed. All animals were monitored for 50 days for tumor formation and then sacrificed when the tumor diameter reached $\sim 1.5 \mathrm{~cm}$. The tumors were resected and tumor volumes were determined using calipers. One part of the tumor specimens was processed for histological analysis and the other was snap-frozen and stored at $-80^{\circ} \mathrm{C}$ for western blot analysis. All animal experiments were undertaken in accordance with the National Institutes of Health Guide for the Care and Use of Laboratory Animals, with the approval of the Scientific Investigation Board of the Second Military Medical University [no: (2008) CTcp008].
Protein extraction and western blot analysis. Primary antibodies against DACH1 (1:1,000) and glyceraldehyde 3-phosphate dehydrogenase (GAPDH) (1:3,000; Santa Cruz Biotechnology, Inc., Santa Cruz, CA, USA) were used. PANC-1 cells and xenograft tumors were lysed in a $10 \mathrm{mM}$ Tris-buffer (pH 7.4) containing $1 \%$ sodium dodecyl sulfate (SDS) and complete protease inhibitors (Roche). Samples of the lysates $(80 \mu \mathrm{g})$ were separated by SDS-polyacrylamide gel electrophoresis, transferred to polyvinylidene fluoride membranes (Millipore, Bedford, MA, USA), and probed with the primary antibodies. After the incubation with horseradish peroxidaseconjugated secondary antibody, the blots were visualized with enhanced chemiluminescence detection reagents (sc-2048) (both from Santa Cruz Biotechnology, Inc.). The intensity of each band was measured using a Fluor-S MultiImager and Quantity One software (Bio-Rad, Hercules, CA, USA).

Statistical analysis. Data are presented as means \pm SD. All statistical calculations were carried out using SPSS 11.7 software (SPSS, Chicago, IL, USA). The Mann-Whitney U test or Student's t-test was used to compare continuous variables between 2 groups. The difference among the means of multiple groups was analyzed by one-way analysis of variance followed by the Tukey's test. Receiver operating characteristics (ROC) curves were established to evaluate the diagnostic potential of miR-194. A P-value $<0.05$ was considered to indicate a statistically significant result.

\section{Results}

Upregulation of miR-194 in PDAC. As we previously described (14), miRNA profiling analysis revealed that among the 713 miRNAs tested, 8 (miR-194*, miR-192*, miR-602, miR-801, miR-194, miR-192, miR-212 and miR-34a*) showed upregulated expression and 8 (miR-923, miR-139-3p, miR-513a-5p, miR-630, miR-30c-1*, miR-887, miR-508-5p and miR-516a-5p) showed downregulated expression in PDAC pools, with a cut-off value of 1.15-fold change. miR-194 showed the greatest changes in expression levels among these differentially expressed miRNAs and thus was further explored for its diagnostic and biological significance in PDAC.

To confirm the microarray results, tissue miR-194 expression was examined by LNA-ISH. Positive cytoplasmic staining for miR-192 was evident in tumor cells but was rarely noted in the stromal cells of the PDAC specimens (Fig. 1A). However, no miR-192 positivity was found in the adjacent normal pancreatic tissues (Fig. 1B).

To confirm the microarray results, miR-194 expression in 31 pairs of PDACs and adjacent normal tissues was examined by qRT-PCR analysis. As shown in Fig. 1C, miR-194 expression was significantly $(\mathrm{P}<0.05)$ elevated in PDACs compared to adjacent normal tissues.

Diagnostic significance of serum miR-194 in PDAC. qRT-PCR analysis revealed that PDAC patients had $>3$-fold greater concentrations of serum miR-194 than duodenal adenocarcinoma patients or healthy controls $(\mathrm{P}<0.05$ for each comparison; Fig. 1D). However, no significant difference in the serum miR-194 level was observed between duodenal adenocarcinoma patients and healthy controls. 

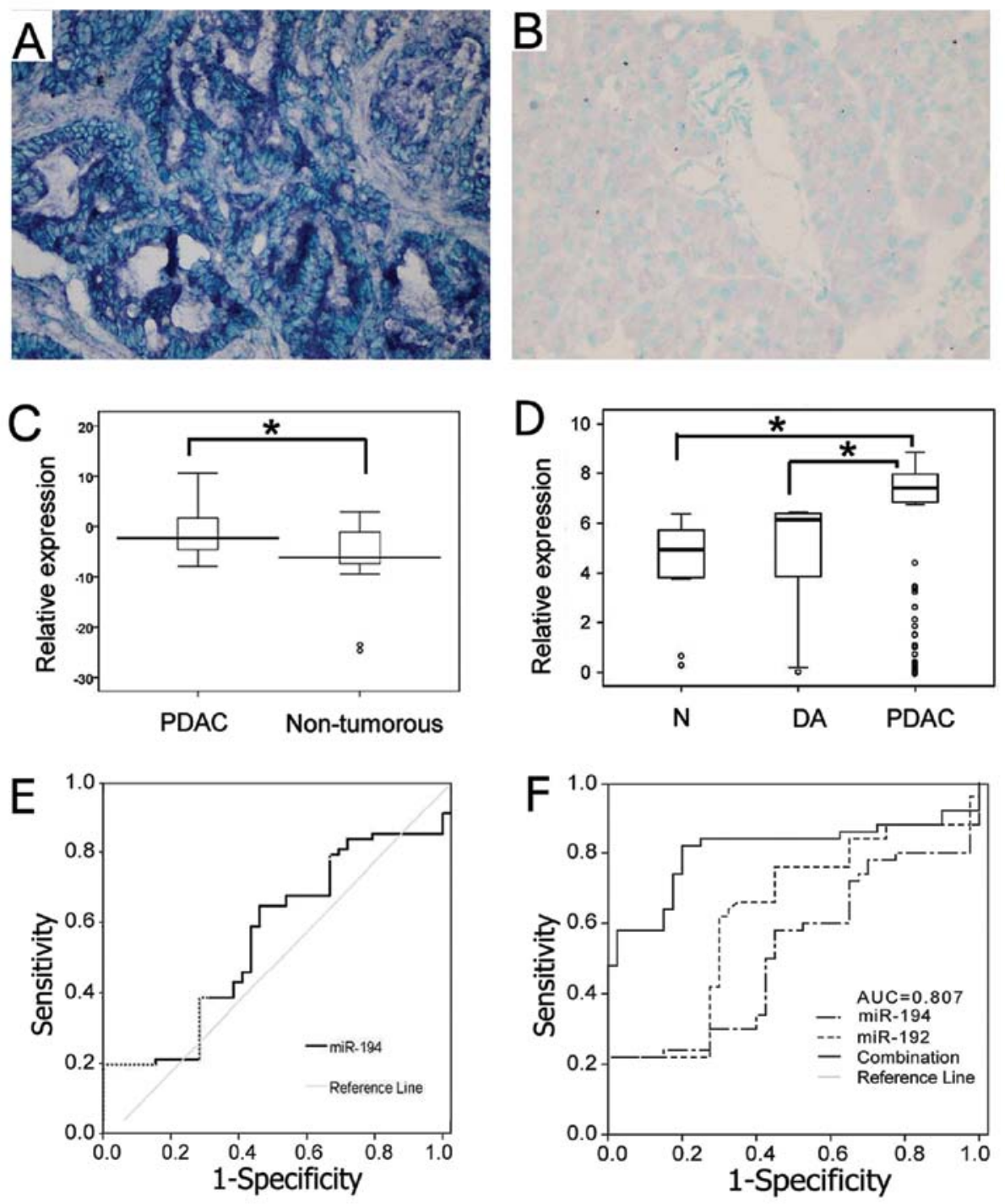

Figure 1. Expression and diagnostic significance of miR-194 in PDAC. Positive cytoplasmic staining for miR-194 was noted in (A) human PDAC tissues but not in (B) adjacent normal pancreatic tissues by LNA-ISH. Original magnification, x400. (C) qRT-PCR analysis of miR-194 levels in 31 pairs of PDACs and adjacent non-tumorous tissues. (D) qRT-PCR analysis of serum miR-194 levels in 70 PDAC patients, 17 age-matched duodenal adenocarcinoma (DA) patients and 40 age-matched normal $(\mathrm{N})$ controls. The relative miRNA expression level was determined by normalizing against U6 snRNA. The thick horizontal line indicates the mean. " $\mathrm{P}<0.05$. (E) ROC curve analysis for discrimination between 70 PDAC patients and 40 healthy controls by serum miR-194. (F) AUCs for miR-192, miR-194 and combination of the 2 were $0.641,0.571$ and 0.807 , respectively. The sensitivity and specificity for miR-192, miR-194 and a combination of the 2 were 76.0 and $55.0 \%, 58.0$ and $55.0 \%$, and 84.0 and $75.0 \%$, respectively. PDAC, pancreatic ductal adenocarcinoma; LNA-ISH, locked nucleic acidbased in situ hybridization; qRT-PCR, quantitative real-time polymerase chain reaction; ROC, receiver operating characteristics; AUCs, area under the curves.

To assess the diagnostic significance of serum miR-194 in differentiating between PDAC patients and healthy individuals, ROC curves were constructed (Fig. 1E). The area under the curve (AUC) was 0.571 [95\% confidence interval (CI), 0.462-0.681]. With an optimal cut-point, the sensitivity and specificity was calculated as 54.3 and $57.5 \%$, respectively.

The possibility of using miR-192 and miR-194 as biomarkers for PDAC diagnosis was examined further. The ROC curves of the miRNAs are presented in Fig. 1F. The area under curve (AUC) was 0.627 (95\% CI, 0.508-0.745) for miR-192 and 0.517 (95\% CI, 0.396-0.638) for miR-194. Analysis of miR-192 and miR-194 together using a discriminant analysis model showed a combined AUC of 0.807 (95\% CI, 0.711-0.903). By means of optimal cut-point determination, the sensitivity and speci- ficity were found to be 76.0 and $55.0 \%$ for miR-192, 58.0 and $55.0 \%$ for miR-194, and 84.0 and $75.0 \%$ for the combination of the 2, respectively (Fig. 1F). Discriminant analysis showed that a combination of miR-192 and miR-194 may be a useful biomarker $(\mathrm{P}<0.01)$ for PDAC diagnosis.

Effects of miR-194 overexpression on the proliferation, colony formation and migration of PANC-1 cells. The relative expression of miR-194 in a panel of pancreatic cancer cell lines was measured by qRT-PCR. We found that miR-194 was the most abundant in AsPC-1 cells, followed by Capan-1, SW1990, BxPC-3, PANC-1 and MIA PaCa-2 cells (Fig. 2A). Similar findings were noted in their culture supernatants (Fig. 2B). Due to the low expression of miR-194, PANC-1 cells were 

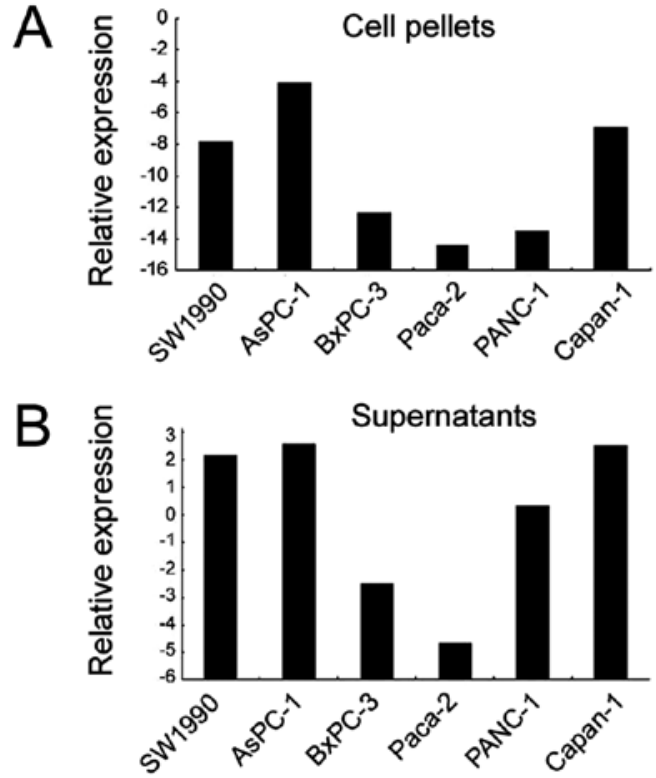

Figure 2. Measurement of miR-194 levels in a panel of (A) human pancreatic cancer lines and their (B) culture supernatants. Bars represent the relative miRNA expression after normalization against U6 snRNA.
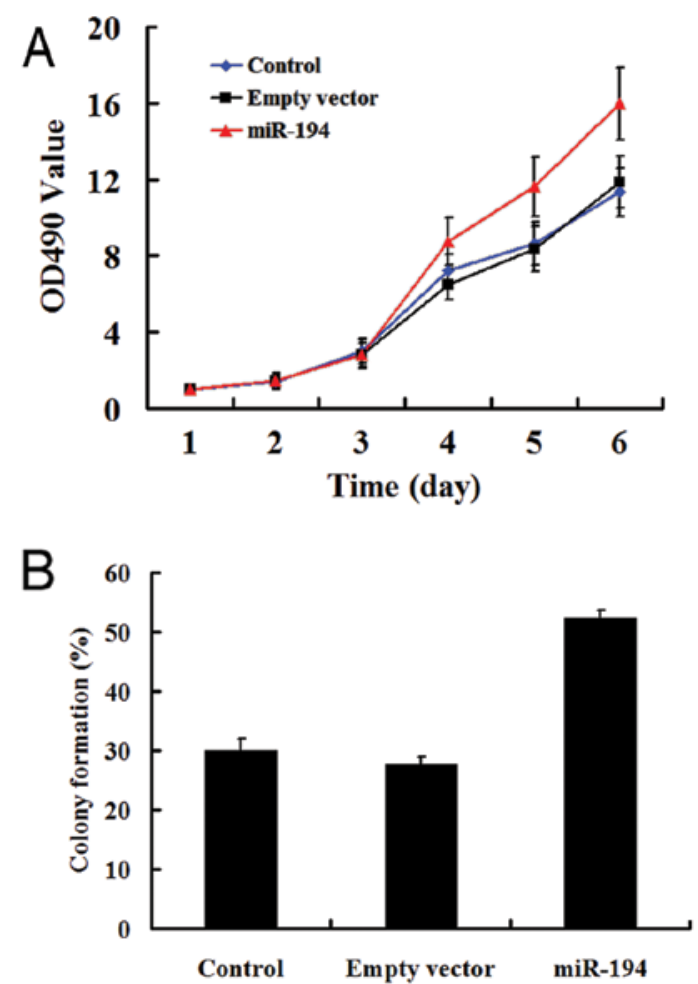

Figure 3. Effects of miR-194 overexpression on the proliferation and colony formation of PANC-1 cells. (A) Evaluation of cell proliferation using the MTT assay. Growth curves were plotted as optical density $(490 \mathrm{~nm})$ vs. time. ${ }^{*} \mathrm{P}<0.05$ vs. empty vector-transduced cells. (B) Colony formation assay revealed a higher colony formation rate for miR-194-overexpressing PANC-1 cells relative to empty vector-transduced cells.

chosen for gain-of-function studies. Lentiviral delivery of miR-194 resulted in a significant increase of miR-194 expression in PANC-1 cells, as compared to control cells transduced with empty lentiviral particles ( $\mathrm{P}<0.05$; data not shown).
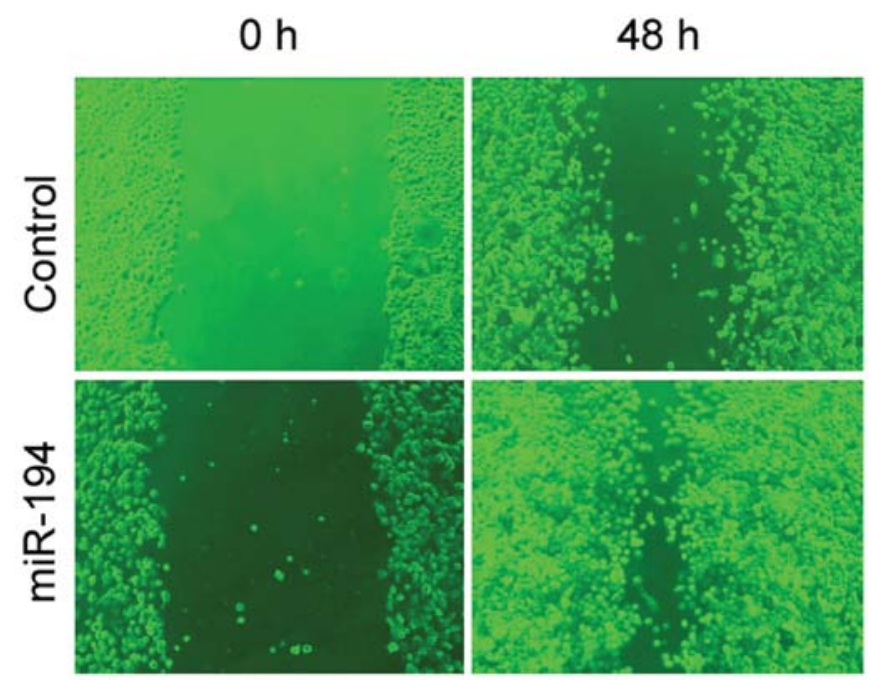

Figure 4. Effects of miR-194 overexpression on the migration in PANC-1 cells. Cell migration assessed by in vitro wound healing assay. Cell monolayers of miR-194-overexpressing and empty vector-transduced PANC-1 cells were scratched and the wound closure was imaged after a 48-h culture period. Representative images of 3 independent experiments are shown.

The MTT assay revealed that miR-194-overexpressing PANC-1 cells had a significant increase in proliferation $72 \mathrm{~h}$ after seeding, as compared to empty lentiviral particle-transduced cells $(\mathrm{P}<0.05$; Fig. 3A). The colony formation assay showed that miR-194-overexpressing PANC-1 cells showed a significantly higher colony formation rate than empty vectortransduced cells $(52.3 \pm 1.3$ vs. $27.7 \pm 1.3 \%, \mathrm{P}<0.01$; Fig. $3 \mathrm{~B})$. In vitro wound healing assay further showed that enforced expression of miR-194 markedly promoted the migration of PANC-1 cells, as evidenced by acceleration of wound closure (Fig. 4).

Effects of miR-194 overexpression on tumorigenicity of PANC-1 cells. Next, we examined the effects of miR-194 overexpression on tumorigenicity of PANC-1 cells, using an orthotopic xenograft model. At 50 days after cell implantation, tumor formation was observed in each animal. Of note, miR-194-overexpressing PANC-1 cells formed significantly larger tumors in nude mice than empty vector-transduced PANC-1 cells $\left(0.4625 \pm 0.127 \mathrm{~cm}^{3}\right.$ vs. $0.196 \pm 0.083 \mathrm{~cm}^{3}$, $\mathrm{P}<0.05$; Fig. 5A and B). Macroscopically, mock-transduced PANC-1 cells dominantly formed small round tumors circumscribed with a thin capsule in nude mice (Fig. 5C). In contrast, xenograft tumors derived from miR-194-overexpressing cells appeared nodular and highly vascularized (Fig. 5D). Histopathologically, miR-194 xenograft tumors comprised a large number of proliferating neoplastic cells and small blood vessels, with local invasion of adjacent muscle, fat and spleen tissues (Fig. 5E-H).

Western blot analysis demonstrated that the DACH1 protein level was profoundly reduced in miR-194 xenograft tumors compared to control tumors (Fig. 6A). In vitro studies confirmed that miR-194-overexpressing PANC-1 cells showed lower DACH1 protein levels than mock-transduced PANC-1 cells (Fig. 6B). 

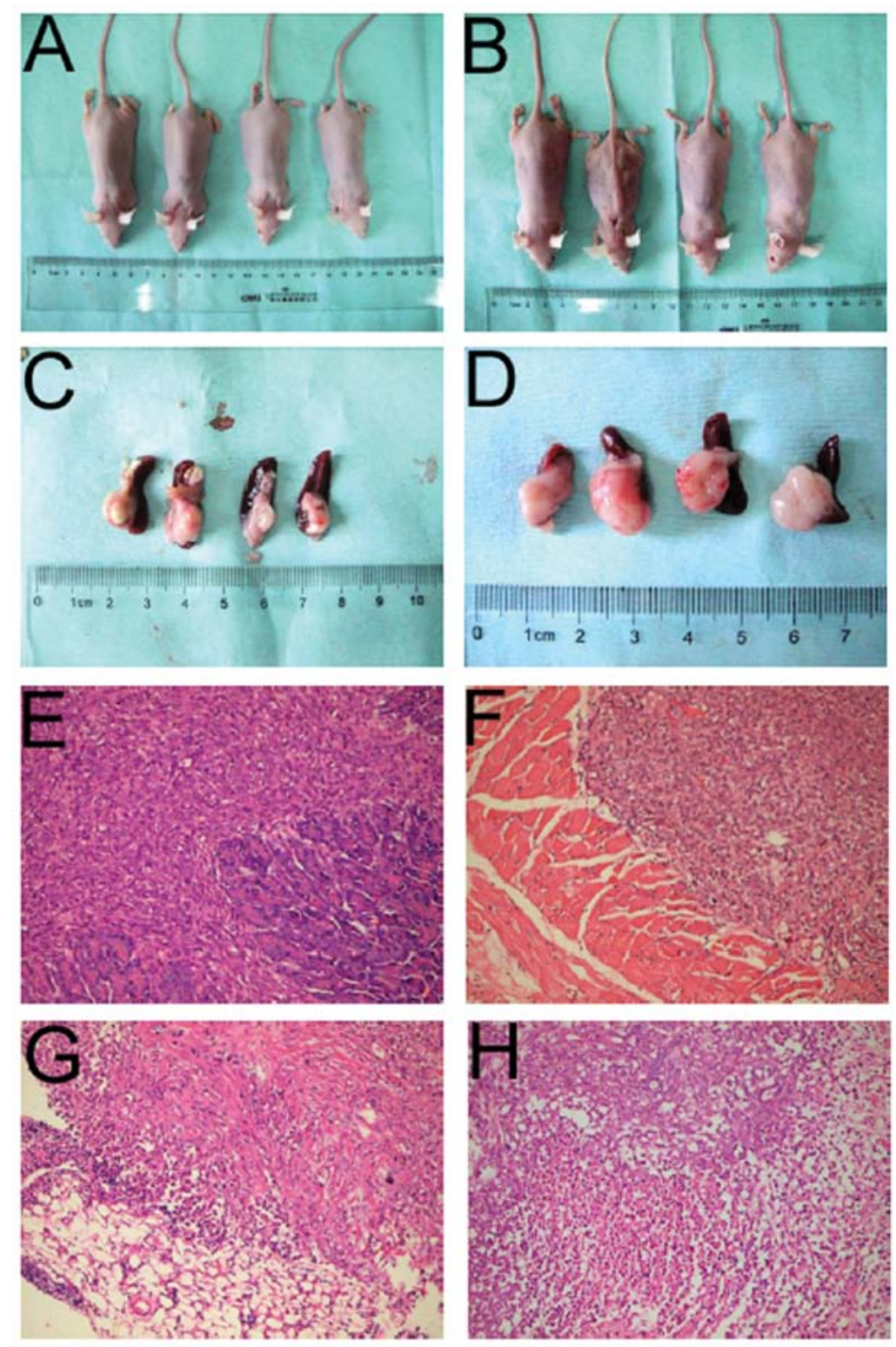

Figure 5. Effects of miR-194 overexpression on tumor growth in a PANC-1 xenograft model. (A) Images of mice bearing tumors in the control and (B) miR-194 groups. Macroscopic images of xenograft tumors from miR-194-overexpressing (D) and empty vector-transduced (C) PANC-1 cells. Hematoxylin and eosin (H\&E) staining analysis revealed that miR-194 xenograft tumors comprised a large number of proliferating neoplastic cells and small blood vessels (E), with local invasion of adjacent muscle $(\mathrm{F})$, fat $(\mathrm{G})$ and spleen tissues $(\mathrm{H})$.

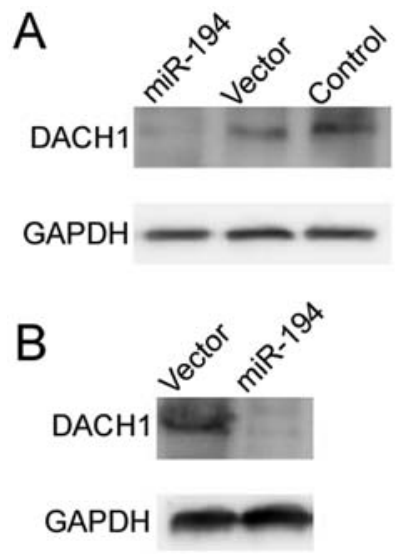

Figure 6. Effects of miR-194 overexpression on DACH1 expression in (A) PANC-1 cells and (B) xenograft tumors. Representative western blotting of 3 independent experiments with similar results are shown. GAPDH was used as a loading control.

\section{Discussion}

Increasing evidence suggests that aberrant expression of miRNAs represents a pathogenic hallmark of cancer (5-7). A large number of miRNAs such as miR-148a, miR-217, miR-196a (17), miR-221 (18) and miR-211 (19) are deregulated in pancreatic cancer and show diagnostic and prognostic relevance in this disease. In the present study, we employed miRNA microarrays to identify novel differentially expressed miRNAs, which may have the potential to be useful biomarkers for cancer detection. Our results revealed that there were 16 miRNAs with $\geq 1.15$-fold expression changes (8 genes overexpressed and 8 underexpressed) in surgically resected PDACs relative to adjacent non-tumorous pancreatic tissues. miR-194 showed the greatest changes in expression levels among these miRNAs and thus was further explored for its diagnostic and biological significance in PDAC. qRT-PCR 
analysis confirmed the microarray findings and revealed that miR-194 was significantly upregulated in PDACs compared to adjacent normal tissues. Similarly, Mees et al (20) reported that upregulation of miR-194 correlates with reduced expression of tumor suppressor EP300 in metastatic PDAC cells. These findings suggest that miR-194 may play a favorable role in PDAC progression.

It has been suggested that some cellular miRNAs can be encapsulated in exosome-like vesicles and released into extracellular environment (13). In supporting this view, our data revealed that miR-194 can be detected in the culture supernatants of a panel of pancreatic cancer cell lines. The mechanism of miRNA release is still not completely understood. A recent study suggested that miRNA release may be mediated through a ceramide-dependent secretory machinery (21). miRNAs are present in human plasma or serum in a markedly stable form that is protected from endogenous RNase activity (22). Therefore, circulating miRNAs have the potential as noninvasive biomarkers for cancer detection. Indeed, circulating miR-378 and miR-451 in serum have been proposed as potential biomarkers for renal cell carcinoma (23). Similarly, miR-221 (18) and miR-18a (18) in the plasma have shown diagnostic significance in pancreatic cancer. Our present data revealed that serum miR-194 concentrations were significantly higher in PDAC patients than in duodenal adenocarcinoma patients and healthy controls. However, serum miR-194 levels may discriminate PDAC patients from healthy individuals with a sensitivity and specificity of only $<60 \%$. The discriminating power indicated by the AUC value (0.571) was markedly lower than the expectations of clinical utility. These findings suggest that serum miR-194 alone may not be suitable as a diagnostic marker for PDAC. Combined analysis of the 3 groups yielded a sensitivity of 84.0 and a specificity of $75.0 \%$ for the combined detection of miR-192 and miR-194 in the diagnosis of PDAC. This indicates that single miRNA may not be a satisfactory tumor marker, but the combined determination of multiple miRNAs can have a high sensitivity and specificity. Wang et al (24) documented that the combination of the 4 miRNAs (miR-21, miR-210, miR-155 and miR-196a) raises the AUC value from 0.62 to 0.69 ranging for each individual miRNA to 0.82 for pancreatic cancer detection. Therefore, the combination of serum miR-194 with other miRNAs or protein biomarkers warrants further study.

Several previous studies suggested that miR-194 often acts as a suppressor of tumor progression and metastasis $(25,26)$. For instance, Khella et al (25) reported that miR-194 is downregulated in metastatic renal cell carcinoma and restoration of its expression decreases cell migration and invasion in renal cell carcinoma cell line models. Similarly, the overexpression of miR-194 in liver mesenchymal-like cancer cells was found to reverse the epithelial-mesenchymal transition and cancer cell invasion and migration (26). However, Iizuka et al (27) showed that miR-194 is upregulated in human breast cancer cell lines and its depletion leads to significant suppression of cell proliferation. Sundaram et al (28) reported that miR-194 overexpression promotes tumor angiogenesis in colon cancer. Therefore, the overall contribution of miR-194 to tumor growth and progression is cellular context dependent. Our results showed that enforced expression of miR-194 promoted cell proliferation, colony formation and migration in PANC-1 cells. The growth-promoting effects of miR-194 overexpression were also observed in the orthotopic pancreatic tumor model. DACH1 functions as a tumor suppressor in several types of cancer, such as breast cancer, glioma and prostate cancer (29-31). Notably, we found that the protein expression of DACH1 was markedly reduced in miR-194-overexpressing PANC-1 cells and xenograft tumors, which provides an explanation for the tumorigenic role of miR-194 in PDAC. Taken together, these findings indicate that miR-194 acts as an oncogene in pancreatic cancer, thus representing a potential therapy target for this disease. However, the exact molecular mechanisms underlying the effects of miR-194 on pancreatic cancer cells needs further elucidation. Additionally, loss-offunction studies are required to complement the results of gain-of-function studies and to further confirm the oncogenic role of miR-194 in PDAC.

In conclusion, miR-194 is upregulated in cancer tissue and serum of patients with PDAC, and serum miR-194 shows a limited capacity for discriminating between PDAC cancer patients and healthy individuals. Combined detection of serum miR-192 and miR-194 level may serve as a sensitive diagnostic biomarker for PDAC. Enforced expression of miR-194 facilitates tumor growth and progression in cell and mouse models of PDAC, possibly through downregulation of the tumor suppressor DACH1. These results facilitate our understanding of the pathogenesis of PDAC and suggest miR-194 as a promising therapeutic target for this disease.

\section{Acknowledgements}

This study was supported by the National Key Project of Scientific and Technical Supporting Programs of China (no. 2006BAI02A14), the National Natural Science Foundation of China (nos. 30770996 and 81172310) to M.H. Zhu; and the ' 085 ' first-class discipline construction of science and technology innovation in Shanghai University of Traditional Chinese Medicine (no. 085ZY1220) to S.H. Zhang.

\section{References}

1. Jemal A, Siegel R, Xu J and Ward E: Cancer statistics, 2010. CA Cancer J Clin 60: 277-300, 2010.

2. Li D, Xie K, Wolff R and Abbruzzese JL: Pancreatic cancer. Lancet 363: 1049-1057, 2004.

3. Brand RE and Matamoros A: Imaging techniques in the evaluation of adenocarcinoma of the pancreas. Dig Dis 16: 242-252, 1998.

4. Yates LA, Norbury CJ and Gilbert RJ: The long and short of microRNA. Cell 153: 516-519, 2013.

5. Fabbri M: MicroRNAs and cancer: towards a personalized medicine. Curr Mol Med 13: 751-756, 2013.

6. Ahmed FE: Role of miRNA in carcinogenesis and biomarker selection: a methodological view. Expert Rev Mol Diagn 7: 569-603, 2007.

7. Cowland JB, Hother $\mathrm{C}$ and Grønbaek K: MicroRNAs and cancer. APMIS 115: 1090-1106, 2007.

8. Madhavan D, Cuk K, Burwinkel B and Yang R: Cancer diagnosis and prognosis decoded by blood-based circulating microRNA signatures. Front Genet 4: 116, 2013.

9. Liffers ST, Munding JB, Vogt M, et al: MicroRNA-148a is down-regulated in human pancreatic ductal adenocarcinomas and regulates cell survival by targeting $C D C 25 B$. Lab Invest 91: 1472-1479, 2011

10. Bhatti I, Lee A, James V, et al: Knockdown of microRNA-21 inhibits proliferation and increases cell death by targeting programmed cell death 4 (PDCD4) in pancreatic ductal adenocarcinoma. J Gastrointest Surg 15: 199-208, 2011. 
11. Liu R, Chen X, Du Y, et al: Serum microRNA expression profile as a biomarker in the diagnosis and prognosis of pancreatic cancer. Clin Chem 58: 610-618, 2012.

12. Munding JB, Adai AT, Maghnouj A, et al: Global microRNA expression profiling of microdissected tissues identifies miR-135b as a novel biomarker for pancreatic ductal adenocarcinoma. Int J Cancer 131: E86-E95, 2012.

13. Ekström K, Bossios A, Sjöstrand M, Lee JJ and Lötvall JO: Exosome-mediated transfer of mRNAs and microRNAs is a novel mechanism of genetic exchange between cells. Nat Cell Biol 9: 654-659, 2007.

14. Zhao C, Zhang J, Zhang S, et al: Diagnostic and biological significance of microRNA-192 in pancreatic ductal adenocarcinoma. Oncol Rep 30: 276-284, 2013.

15. Rabinowits G, Gerçel-Taylor C, Day JM, Taylor DD and Kloecker GH: Exosomal microRNA: a diagnostic marker for lung cancer. Clin Lung Cancer 10: 42-46, 2009.

16. Livak KJ and Schmittgen TD: Analysis of relative gene expression data using real-time quantitative PCR and the $2^{-\Delta \Delta C_{\mathrm{T}}}$ method Methods 25: 402-408, 2001.

17. Xue Y, Abou Tayoun AN, Abo KM, et al: MicroRNAs as diagnostic markers for pancreatic ductal adenocarcinoma and its precursor, pancreatic intraepithelial neoplasm. Cancer Genet 206: 217-221, 2013

18. Kawaguchi T, Komatsu S, Ichikawa D, et al: Clinical impact of circulating miR-221 in plasma of patients with pancreatic cancer. Br J Cancer 108: 361-369, 2013.

19. Giovannetti E, van der Velde A, Funel N, et al: High-throughput microRNA (miRNAs) arrays unravel the prognostic role of miR-211 in pancreatic cancer. PLoS One 7: e49145, 2012.

20. Mees ST, Mardin WA, Wendel C, et al: EP300 - a miRNAregulated metastasis suppressor gene in ductal adenocarcinomas of the pancreas. Int J Cancer 126: 114-124, 2010.

21. Kosaka N, Iguchi H, Yoshioka Y, Takeshita F, Matsuki Y and Ochiya T: Secretory mechanisms and intercellular transfer of microRNAs in living cells. J Biol Chem 285: 17442-17452, 2010.
22. Mitchell PS, Parkin RK, Kroh EM, et al: Circulating microRNAs as stable blood-based markers for cancer detection. Proc Natl Acad Sci USA 105: 10513-10518, 2008.

23. Redova M, Poprach A, Nekvindova J, et al: Circulating miR-378 and miR-451 in serum are potential biomarkers for renal cell carcinoma. J Transl Med 10: 55, 2012.

24. Wang J, Chen J, Chang P, et al: MicroRNAs in plasma of pancreatic ductal adenocarcinoma patients as novel blood-based biomarkers of disease. Cancer Prev Res 2: 807-813, 2009.

25. Khella HW, Bakhet M, Allo G, et al: miR-192, miR-194 and miR-215: a convergent microRNA network suppressing tumor progression in renal cell carcinoma. Carcinogenesis 34: 2231-2239, 2013

26. Meng Z, Fu X, Chen X, et al: miR-194 is a marker of hepatic epithelial cells and suppresses metastasis of liver cancer cells in mice. Hepatology 52: 2148-2157, 2010.

27. Iizuka D, Imaoka T, Nishimura M, Kawai H, Suzuki $F$ and Shimada Y: Aberrant microRNA expression in radiationinduced rat mammary cancer: the potential role of miR-194 overexpression in cancer cell proliferation. Radiat Res 179: 151-159, 2013.

28. Sundaram P, Hultine S, Smith LM, et al: p53-responsive miR-194 inhibits thrombospondin-1 and promotes angiogenesis in colon cancers. Cancer Res 71: 7490-7501, 2011.

29. Popov VM, Zhou J, Shirley LA, et al: The cell fate determination factor DACH1 is expressed in estrogen receptor- $\alpha$-positive breast cancer and represses estrogen receptor- $\alpha$ signaling. Cancer Res 69: 5752-5760, 2009.

30. Watanabe A, Ogiwara H, Ehata S, et al: Homozygously deleted gene DACH1 regulates tumor-initiating activity of glioma cells. Proc Natl Acad Sci USA 108: 12384-12389, 2011.

31. Wu K, Katiyar S, Witkiewicz A, et al: The cell fate determination factor dachshund inhibits androgen receptor signaling and prostate cancer cellular growth. Cancer Res 69: 3347-3355, 2009. 